\title{
MEJORAMIENTO DE LAS PROPIEDADES MECÁNICAS DEL POLIPROPILENO RECICLADO MEDIANTE ADICIÓN DE FIBRAS VEGETALES, POLIETILEN TERFTALATO Y TRATAMIENTO TÉRMICO.
}

\section{Improvement of the mechanical properties of recycled polypropylene through addition of vegetable fibers, polyethylene terphthalate and heat treatment.}

\author{
${ }^{1}$ Paul Palmay* (D), ${ }^{1}$ Sofía Sanipatin (iD, ${ }^{1}$ Daniela Poalacin (iD, ${ }^{2}$ Caterine Donoso
}

${ }^{1}$ Escuela Superior Politecnica de Chimborazo, Facultad de Ciencias, Riobamba, Ecuador. ${ }^{2}$ Universidad Tecnica de Cotopaxi, Facultad de Ciencias Agropecuarias y Recursos Naturales, Latacunga, Ecuador.

*paul.palmay@espoch.edu.ec

$\mathrm{R}$ esumen

En los residuos sólidos plásticos el polipropileno es uno de los termoplásticos de mayor consumo a nivel urbano y por ende el de mayor generación de residuos. En este sentido, el reciclaje mecánico es la tecnica mas sencilla de aplicación aprovechando de residuos de la misma familia de termoplastico que acondicionado con otras fibras permiten el mejoramiento de sus propiedades, mediante la elaboración de composites con fibras naturales o sinteticas. El presente estudio tiene como objetivo el analisis de las propiedades más importantes de composites hechos de poliproplieno con fibras naturales: de plátano y de abacá; composites de polipropileno con polietilen tereftalato a diferentes concentraciones, con y sin acoplante; y un tratamiento termico para el mejoramiento de la matriz de polipropileno reciclado. Se obtienen probetas mediante extrusión de monohusillo a diferentes perfiles de temperatura y concentraciones de fibra adicionada. Se plantea el tratamiento térmico al polipropileno midiendo el grado de cristalización que se genera. Posterior, se mide la tracción, flexión y calorimetría diferencial de barrido de los composites de buena trabajabilidad usando como acoplante anhidrido terftalico. Los resultados muestran mejoras en las propiedades de tracción y flexión al momento de adición del agente acoplante en ambos composites y con concentraciones bajas de fibra de refuerzo.

Palabras claves: Reciclaje Mecánico, Plásticos Post consumo, Compostes Polipropileno.

\section{A bstract}

In solid plastic waste, polypropylene is one of the thermoplastics with the highest consumption in urban areas and, therefore, the one with the highest waste generation. In this sense, mechanical recycling is the simplest application technique taking advantage of the waste of the same family of thermoplastics that conditioned with other fibers allow the improvement of their properties, through the elaboration of composites with natural or synthetic fibers. This study aims to analyze the most important properties of compounds made of polypropylene with natural fibers: banana and abaca; polypropylene compounds with polyethylene terephthalate in different concentrations, with and without couplant; and a heat treatment for the improvement of the recycled polypropylene matrix. Samples are obtained by single screw extrusion at different temperature profiles and concentrations of added fiber. The heat treatment of polypropylene is proposed by measuring the degree of crystallization that is generated. Subsequently, the calorimetry of traction, flexion and differential sweep of good workability compounds is measured using terphthalic anhydrous coupler. The results show improvements in tensile and bending properties at the time of addition of the coupling agent in both compounds and with low concentrations of reinforcing fiber.

Keywords: Mechanical Recycling, Post-consumer Plastics, Polypropylene Composts 


\section{INTRODUCCIÓN}

En la actualidad, la tecnología ha orientado sus esfuerzos en combinar las propiedades de diferentes tipos de materiales, a fin de obtener materiales compuestos con mejores propiedades o con propiedades útiles para aplicaciones específicas.

El reciclaje de termoplásticos reforzados con fibra natural recibe gran atención debido a su bajo costo, buenas propiedades y en especial a su biodegradabilidad, razón que las convierte en un reemplazo adecuado al uso de fibras sintéticas usadas en el sector corporativo de los polímeros $(1,2)$.

Las propiedades de la matriz, las fibras y la interfaz fibra-matriz cambian con cada reciclaje siendo de suma importancia tomar en cuenta la relación (L / D) de las fibras, que representa la relación entre la longitud (L) y el diámetro (D) de las fibras de refuerzo (3), ya que de acuerdo a esto se evaluaran las propiedades mecánicas obtenidas del composite.

Este parámetro es de suma importancia ya que ofrece una idea clara de la unión interfacial, así como la transferencia de carga entre las fibras y la matriz de plástico. Existen varios estudios de refuerzo con fibras naturales como cáñamo o sisal, donde se presenta un mejoramiento de la matriz de plástico en sus propiedades mecánicas de los composites $(4,5)$.

La combinación de diferentes polímeros con la matriz base de propileno mejora ciertas propiedades físicas como la resistencia a la tracción y la flexión, entre estos tenemos el tereftalato de polietileno (PET).

Las matrices poliméricas obtenidas se utilizan cada vez más para reemplazar piezas metálicas debido, entre otras cosas, a una mejor relación resistencia / densidad y bajo costo $(6,7)$. Las fibras de refuerzo pueden variar entre sintéticas por ejemplo el Kevlar o Twaron (8), o fibras naturales provenientes de biomasas fibrosas que mejoran las propiedades principalmente de flexión a los composites con polipropileno reciclado $(7,9)$. Sin embargo el principal problema de la adición de
PET a la matriz de PP es la inmiscibilidad de sus estructuras, razón por lo cual es importante la disminución de la tensión superficial entre los polímeros mediante el uso de un agente acoplante que disminuya la tensión superficial entre las fibras y la matriz consiguiendo su incorporación $(10,11)$.

El presente estudio analiza dos metodologías de reciclaje de polipropileno: refuerzo con fibra natural y refuerzo con PET reciclado en los que se usa agente acoplante no convencional denominado anhídrido tereftálico para mejorar la interfaz entre el lado apolar de los refuerzos y el lado polar de la matriz de PP. Adicional se prueba con el mejoramiento de sus propiedades mediante tratamiento térmico como técnica adicional a las convencionales, utilizando plásticos post consumo.

\section{MATERIALES Y MÉTODOS}

Para la obtención de las probetas de PP se usa una extrusora tipo husillo de $4983 \mathrm{~mm}$ de largo con un tornillo de diámetro de $50,9 \mathrm{~mm}$ y de 9 filetes a lo largo de este, calentado por tres resistencias eléctricas las cuales se controlan con 4 termocuplas en las diferentes zonas.

A la salida de la boquilla se enfría con un baño de agua y un posterior prensado para moldeo a presión de 3500 psi. Adicional cuenta con un variador de frecuencia para el control de las revoluciones de giro. Obteniendo probetas tipo A que están elaboradas con $\mathrm{PP}$ reciclado y tipo B composites de PET y fibra vegetal con y sin acoplante, como se muestra en las figuras 1 y 2 , en donde se representa las diferentes longitudes de la probeta con l, el ancho de la probeta en la zona de prueba como bl y h como el espesor de la probeta.

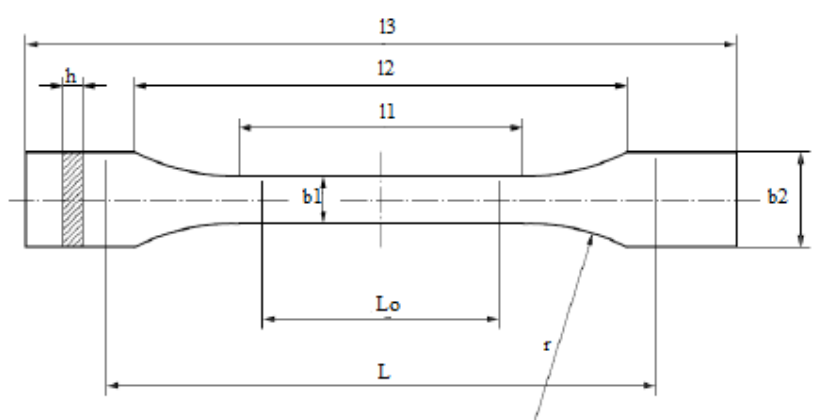

Figura 1. Molde de probeta Tipo B para plástico reforzado con fibra vegetal. 


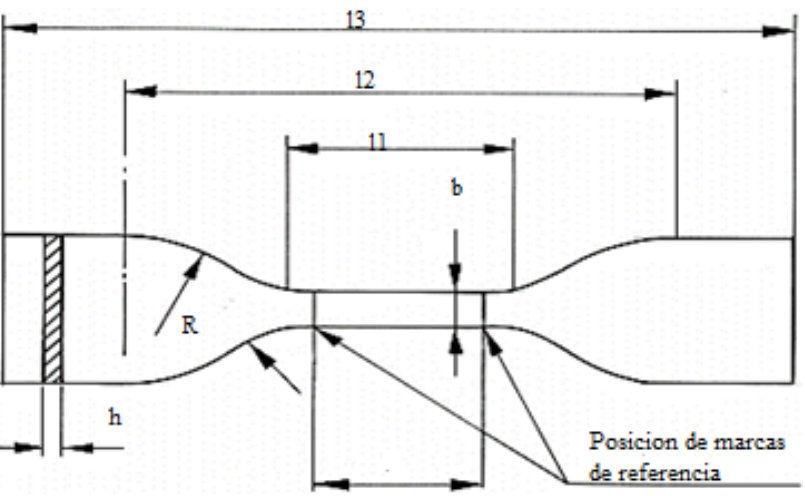

Figura 2. Probeta tipo A para ensayos de tracción en plástico en composites.

Fuente: Norma International ISO 527-2:2012. Plastics -- Determination of tensile properties -- Part 2: Test conditions for mounding and extrusion plastics.

Para el refuerzo con fibra natural, se trabajó con fibra de Abacá (Musa textiles) o también llamado cáñamo de manila y con fibra de Plátano (Musa $\mathrm{AAB}$ ) proveniente del pseudotallo, variando los porcentajes en el rango de 10 a 30\% de fibra. Mientras que para el tratamiento térmico mediante la temperatura de transición vítrea del PP reciclado se calculó la temperatura de recocido, teniendo la precaución de que la estabilidad dimensional de la pieza se preserve y que al mismo tiempo no aparezcan fenómenos de degradación térmica $(12,13)$.

\section{ELABORACIÓN DEL MATERIAL COM- PUESTO CON FIBRA VEGETAL}

El abacá y el plátano pertenecen a la familia de las musáceas y poseen propiedades en común, razón por la cual se somete al mismo tratamiento ambos tipos de fibra. El cual consiste en una separación de sus vainas mediante un desfibrado manual, secado al sol y un posterior corte de la fibra hasta un tamaño de $5 \mathrm{~mm}$ de largo aproximadamente ya que con este tamaño de partícula se consigue una distribución de fibra en la matriz adecuada el momento de la extrusión. Las proporciones en que se empleó las fibras son 10 , 20 y $30 \%$ de fibra, en tratamientos con $1 \%$ de acoplante y sin acoplante.

Este método de procesamiento y preparación de los composites aseguró una buena distribución de fibra y una relación apropiada entre fibra y matriz que otorgó las propiedades mecánicas esperadas. La orientación en paralelo de las fibras asegura la homogeneidad en cuanto a distribu- ción de sus componentes y de las propiedades mecánicas resultantes. Sin embargo al usarse fibras naturales que contiene lignocelulosa se debe prestar atención en mantener la temperatura de procesamiento relativamente baja $\left(200^{\circ} \mathrm{C}\right)$ para evitar la descomposición de la fibra y la disminución de la resistencia de las fibras (8), razón por la que se determinó las condiciones de temperatura en las diferentes zonas y la velocidad de giro del tornillo que permitan una extrusión adecuada.

Para ello se trabajó con tres variantes en las temperaturas del tornillo, estimadas en el rango de 160 a $200^{\circ} \mathrm{C}$, datos sugeridos en estudios previos similares al presente trabajo. Tomando en cuenta como factor determinante la facilidad de trabajo en el proceso de extrusión y el grado de fluidez que toma el PP en conjunto con la fibra sin quemarse para obtener las probetas en las cuales se realizará las pruebas físico mecánicas.

El tornillo extrusor fue operado a una temperatura promedio de $185^{\circ} \mathrm{C}$ en la zona de dosificación, en la zona de transición y compresión a una temperatura promedio de $175^{\circ} \mathrm{C}$ y en la zona de alimentación a una temperatura promedio de $162{ }^{\circ} \mathrm{C}$, variando la velocidad del tornillo entre 80 a $500 \mathrm{rpm}$ en rangos de 50 hasta conseguir una presión de extrusión adecuada para cada tratamiento(14).

\section{ELABORACIÓN DEL MATERIAL COM- PUESTO CON PET}

Para la preparación de las mezclas con PET se plantea un tratamiento variando $5,10,20$ y 30 $\%$ de PET, acorde a lo expuesto por $(15,16)$ y un segundo tratamiento con una concentración al 1 , 3 y $5 \%$ de anhídrido ftálico en polvo dentro de cada mezcla y sin acoplante.

De forma similar el perfil de temperatura para el composite con PET se definió en base a los puntos de fusión del polipropileno, polietileno tereftalato y el compatibilizador (anhídrido ftálico). El tornillo extrusor fue operado a una temperatura promedio de $190{ }^{\circ} \mathrm{C}$ en la zona de dosificación, transición y compresión y en la zona de alimentación a una temperatura promedio de 155 ${ }^{\circ} \mathrm{C}(10,15)$. 
TRATAMIENTO TÉRMICO DE POLIPROPILENO RECICLADO

Se trabajó con dos temperaturas de recocido 125 y $135^{\circ} \mathrm{C}$, que se determinaron a partir de la temperatura de transición vítrea variando el tiempo de recocido de las probetas. La temperatura de la boquilla fue de $160^{\circ} \mathrm{C}$.

\section{RESULTADOS}

\section{PROPIEDADES FÍSICAS POLIPROPILENO Y POLIETILENO TEREFTALATO}

Las muestras se denominan como B1, B2, B3 y B4 que corresponden a las composiciones de PET añadido en la matriz de PP, al 5, 10, 20 y $30 \%$ de PET respectivamente. Mientras que se denominan como $\mathrm{C} 1, \mathrm{C} 2$ y $\mathrm{C} 3$ las probetas que corresponden a las composiciones de acoplante añadido en la matriz de PP/PET, al 1, 2, $3 \%$ de PET respectivamente.

\section{ENSAYO DE TRACCIÓN}

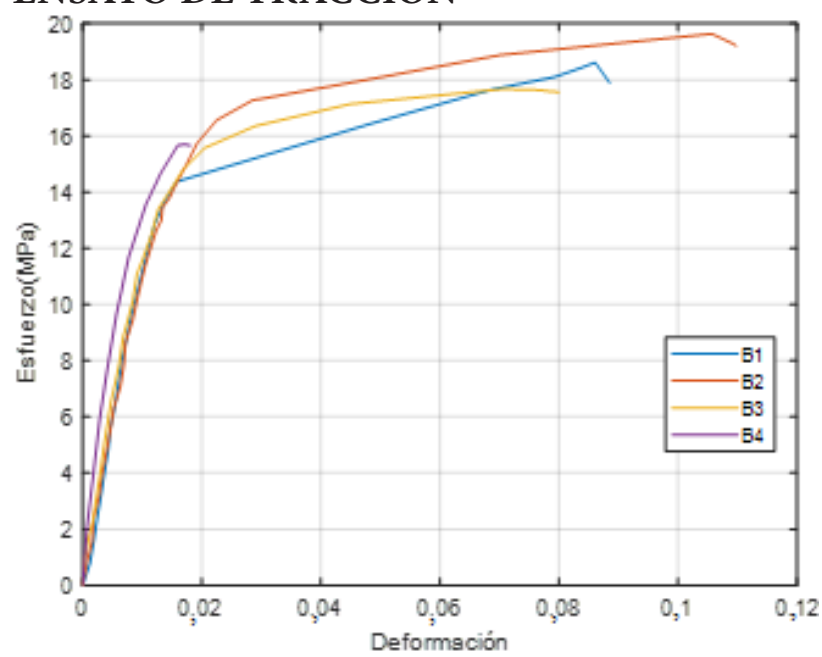

Figura 3. Esfuerzo vs deformación mezclas PP y PET sin acoplante.

En la Figura 3 y 4 . se presenta las curvas de resistencia a los esfuerzos de tracción, para refuerzo tanto con fibra como con PET reciclado con y sin acoplante, donde se puede ver que las de tipo C presentan un mayor esfuerzo máximo esto debido a que el acoplante fortalece el composite al ayudar en la interfaz entre el PP y el PET y aumenta su resistencia consiguiendo valores cercanos a los $20 \mathrm{MPa}(14,17)$.

En cuanto a la figura 3 donde se presenta los composites sin acoplante se puede observar que la mezcla que da mejores propiedades en este ensayo es la correspondiente a la mezcla de $10 \%$ PET y $90 \% \mathrm{PP}$; mientras que en la figura 4 se puede observar que las probetas con acoplante presenta mayor resistencia con la composición de 5\% PET y $95 \% \mathrm{PP}$, lo que quiere decir que cuando añadimos acoplante cambia el comportamiento a tracción de las mezclas, el PP mejora las propiedades de la mezcla por el fortalecimiento de la matriz debido al entrecruzamiento de los componentes en la estructura gracias a los puntos de anclaje que proporciona el agente acoplante llegando a una saturación máxima luego de una cantidad determinada de PET en este caso el 10\% (18-20).

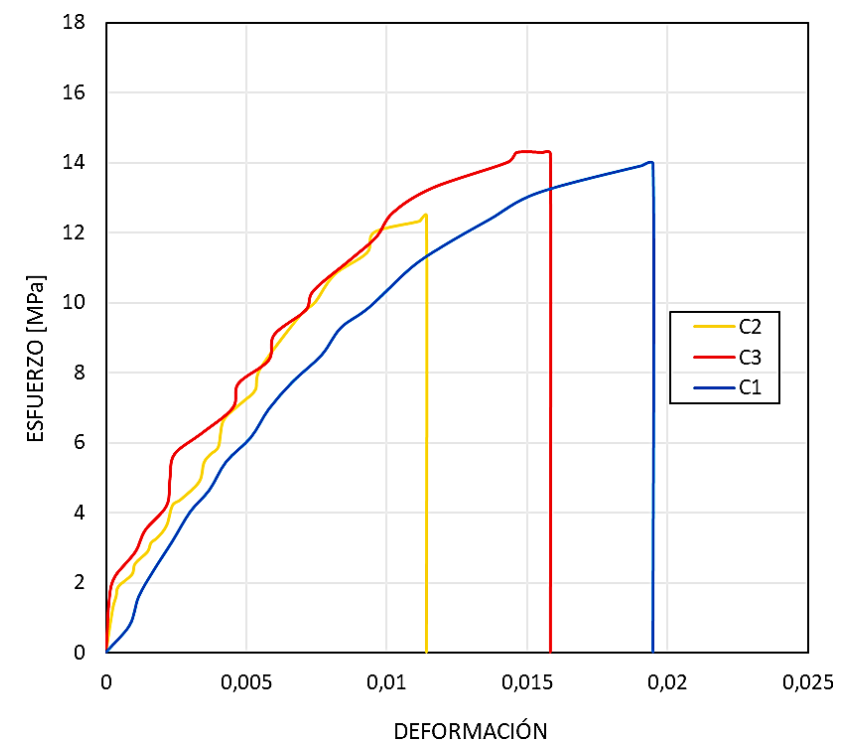

Figura 4. Esfuerzo vs Deformación mezclas PP y PET con acoplante.

\section{ENSAYO DE FLEXIÓN}

\begin{tabular}{|c|c|c|c|c|c|c|c|}
\hline & \multicolumn{7}{|c|}{ MEZCLAS EXTRUIDAS } \\
\hline & B1 & B2 & B3 & B4 & $\mathrm{C} 1$ & $\mathrm{C} 2$ & $\mathrm{C} 3$ \\
\hline $\begin{array}{l}\text { Modulo } \\
\text { Aparente de } \\
\text { Elasticidad } \\
{[\text { MPa] }}\end{array}$ & 1708,5 & 1708,5 & 1708,5 & 1708,5 & 1750,4 & 1184,6 & 2908,9 \\
\hline $\begin{array}{l}\text { Esfuerzo } \\
\text { en la rotura } \\
\text { [MPa] }\end{array}$ & 30,1 & 24,9 & 31,1 & 33,7 & 33,3 & 32,2 & 32,0 \\
\hline
\end{tabular}

Tabla 1. Resultados del ensayo de flexión de las mezclas extruidas.

Como se presenta en la tabla 1, la presencia de mayor cantidad de PET conjugado con la presencia de agente acoplante que permite la mejor distribución del refuerzo en la matriz de PP mejora la propiedad de flexión esto debido a las características en si del PET y que se incorporan al composite $(6,21)$. 
PROPIEDADES FÍSICAS POLIPROPILENO Y FIBRA DE ABACÁ

\begin{tabular}{ccccccccc}
\hline A & B & C & D & E & F & G & H & I \\
\hline PP & \multicolumn{4}{c}{ ABACÁ } & \multicolumn{6}{c}{ PLÁTANO } \\
\hline \multicolumn{4}{c}{$10 \%$} & \multicolumn{2}{c}{$20 \%$} & \multicolumn{2}{c}{$10 \%$} & \multicolumn{2}{c}{$20 \%$} \\
\multicolumn{2}{c}{ s/a } & c/a & s/a & c/a & s/a & c/a & s/a & c/a \\
\hline
\end{tabular}

Tabla 2. Nomenclatura para las muestras empleadas.

s/a: sin acoplante

c/a con acoplante

\section{ENSAYO DE TRACCIÓN}

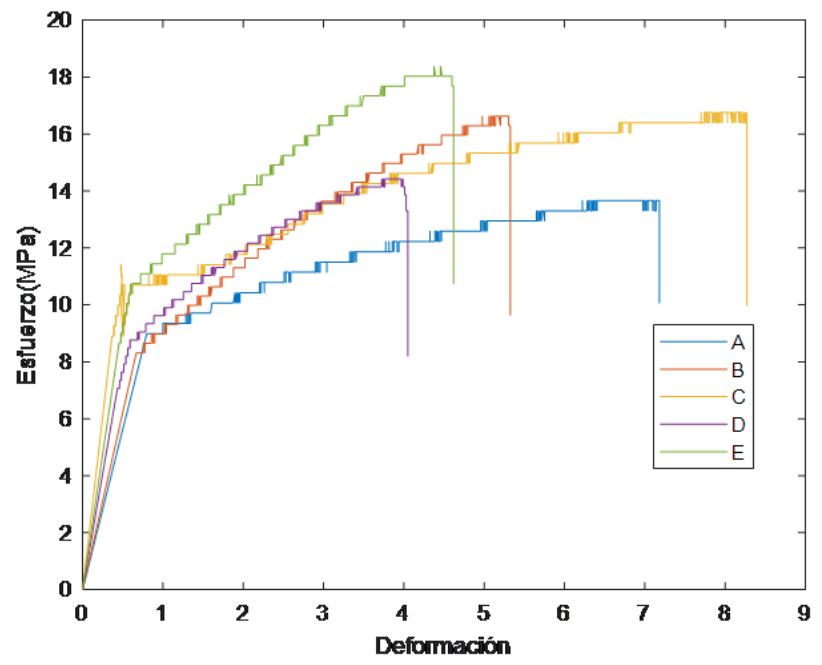

Figura 5. Resultados del ensayo de Tracción PP-Fibra.

De la figura 5 se puede observar que cuando tenemos una mezcla de $10 \%$ de Abacá y $90 \%$ de polipropileno el material compuesto presenta mayor deformación, debido a que las fibras tienen un comportamiento frágil al esfuerzo, pero una matriz dúctil $(5,22)$.

\section{ENSAYO DE FLEXIÓN}

\begin{tabular}{cccccc}
\hline Pruebas & Unidad & $\mathrm{B}$ & $\mathrm{C}$ & $\mathrm{D}$ & $\mathrm{E}$ \\
\hline $\begin{array}{c}\text { Módulo de elasti- } \\
\text { cidad }\end{array}$ & $\mathrm{MPa}$ & 1119,81 & 2005,84 & 677,47 & 1767,37 \\
\hline Esfuerzo de Rotura & $\mathrm{MPa}$ & 16,30 & 41,39 & 21,54 & 29,55 \\
\hline Flecha en la rotura & $\mathrm{mm}$ & 24,56 & 14,52 & 7,77 & 8,60 \\
\hline
\end{tabular}

A partir de la Tabla 2. se puede observar que cuando se trabaja con una mezcla de $10 \%$ de Abacá y $90 \%$ de polipropileno (C) el material compuesto alcanza su mayor flexibilidad, ya que la fibra es altamente flexible y combina estas propiedades con las de la matriz obteniendo un material que en un gran porcentaje aumenta su flexibilidad(23).

\section{FIBRA DE PLÁTANO}

\section{ENSAYO DE TRACCIÓN}

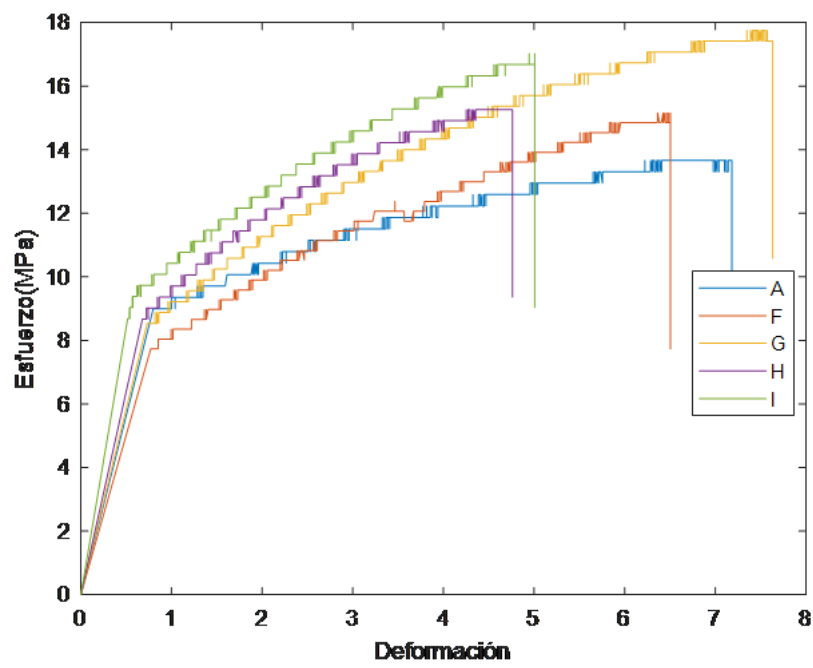

Figura 6. Resultados del ensayo de Tracción en mezcla PP-Fibra.

De la figura 6 se puede observar que cuando tenemos una mezcla $(\mathrm{F})$ de $10 \%$ de fibra de plátano y $90 \%$ de polipropileno el material compuesto alcanza su mayor ductilidad, mejorando la ductilidad que presenta la matriz esto debido a que el material combina las propiedades de la matriz con la fibra.

\section{ENSAYO DE FLEXIÓN}

En cuanto a la propiedad de flexión de las muestras ensayadas se tiene que la mezcla de $10 \%$ de fibra de plátano y $90 \%$ de polipropileno el material compuesto alcanza su mayor flexibilidad, ya que la fibra es altamente flexible y combina estas propiedades con las de la matriz obteniendo un material que en un gran porcentaje aumenta su flexibilidad. Tanto del material compuesto con fibra de abacá y fibra de plátano podemos observar que las mejores propiedades se obtienen cuando tenemos un $10 \%$ de fibra y $90 \%$ de matriz $(5,16,21)$.

\section{PROPIEDADES FÍSICAS CON TRATAMIEN- TO TÉRMICO}

Realizadas las pruebas mecánicas a las probetas con recocido se muestra que la temperatura de $135{ }^{\circ} \mathrm{C}$ presenta buenos resultados mediante ambos enfriamientos con agua y con aire. Sin embargo, el enfriamiento con agua presenta una 
pérdida del $54,4 \%$ en promedio del módulo de elasticidad debido al que enfriamiento brusco que genera matrices más rígidas $(24,25)$. A pesar de esto al haber sido tratada a la misma temperatura ambas muestras presentan una entalpia de 55 y $56 \mathrm{~J}$ g-1 respectivamente, así como un porcentaje de cristalización de 33 y $34 \%$, que comparado con el $24 \%$ medido a las muestras iniciales de polipropileno reciclado presentan un considerable mejoramiento por la disminución de las zonas amorfas de la estructura.

\section{CONCLUSIONES}

Las curvas de esfuerzo respecto a la deformación para el material compuesto desarrollado, en general presentan un comportamiento frágil ante la tensión, pero dúctil por su matriz polimérica; lo que está relacionado con una posible alineación longitudinal de las fibras en los casos en que se presenta mayor proporción de estas. Evidenciándose el mejoramiento de las propiedades del polipropileno reciclado al añadir un composite sea PET o sea fibra vegetal. Aumentando esta tendencia el momento de la adición de acoplante ya que genera una mejor distribución de las fi- bras

Al realizar las diferentes pruebas mecánicas como son resistencia a la tracción, flexión y compresión a las probetas de PP reciclado y las injertadas con fibra obtenidos por extrusión, es notoria la incidencia de la fibra en los resultados en comparación al PP, en los ensayos de resistencia a la tracción mejora el esfuerzo de fluencia un $22 \%$ al trabajar con fibra de abacá con y sin acoplante, ensayos de flexión mejoran únicamente las propiedades al trabajar con $20 \%$ de fibra y uso de acoplante, mejorando las propiedades físicas y mecánicas de las probetas en forma general al trabajar con fibra de abacá y agente acoplante, esto debido a que el anhídrido ftálico promueve la adhesión entre la matriz de PP y cargas inorgánicas mediante la formación de enlaces químicos estables entre la matriz orgánica y la superficie de la carga.

En cuanto, al tratamiento térmico realizado al polipropileno, mejora las propiedades de cristalinidad del PP debido a su re estructuración con la temperatura generando mayor dureza pero menor resistencia a la flexión.

$\mathrm{R}$ eferencias

1. Sullins T, Pillay S, Komus A, Ning H. Hemp fiber reinforced polypropylene composites: The effects of material treatments. Compos Part B Eng [Internet]. 2017;114:15-22. Available from: http:// dx.doi.org/10.1016/j.compositesb.2017.02.001

2. Shrivastava A. Environmental Aspects of Plastics. Introd to Plast Eng. 2018;207-32.

3. Gu F, Guo J, Zhang W, Summers PA, Hall P. From waste plastics to industrial raw materials: A life cycle assessment of mechanical plastic recycling practice based on a real-world case study. Sci Total Environ. 2017;601-602:1192-207.

4. Lila MK, Singhal A, Banwait SS, Singh I. A recyclability study of bagasse fiber reinforced polypropylene composites. Polym Degrad Stab [Internet]. 2018;152:272-9. Available from: https://doi. org/10.1016/j.polymdegradstab.2018.05.001

5. Senthilkumar K, Saba N, Rajini N, Chandrasekar M, Jawaid M, Siengchin S, et al. Mechanical properties evaluation of sisal fibre reinforced polymer composites: A review. Constr Build Mater [Internet]. 2018;174:713-29. Available from: https://doi.org/10.1016/j.conbuildmat.2018.04.143

6. Matias ÁA, Lima MS, Pereira J, Pereira P, Barros R, Coelho JFJ, et al. Use of recycled polypropylene/poly(ethylene terephthalate) blends to manufacture water pipes: An industrial scale study. Waste Manag. 2020;101:250-8.

7. Bommanna K, Shanthakumar GC. Study of Sisal Fibre Attrition and Characterization during Direct Extrusion Compression Moulding. Mater Today Proc [Internet]. 2018;5(5):13251-7. Available from: https://doi.org/10.1016/j.matpr.2018.02.316

8. Bledzki AK, Franciszczak P, Osman Z, Elbadawi M. Polypropylene biocomposites reinforced with softwood, abaca, jute, and kenaf fibers. Ind Crops Prod [Internet]. 2015;70:91-9. Available from: http://dx.doi.org/10.1016/j.indcrop.2015.03.013 
9. Dickson AR, Sandquist D. Mode of wood fibre breakage during thermoplastic melt processing. Compos Part A Appl Sci Manuf [Internet]. 2018;112(July):496-503. Available from: https://doi. org/10.1016/j.compositesa.2018.07.004

10. Nonato RC, Bonse BC. A study of PP/PET composites: Factorial design, mechanical and thermal properties. Polym Test [Internet]. 2016;56:167-73. Available from: http://dx.doi.org/10.1016/j. polymertesting.2016.10.005

11. Saba N, Paridah MT, Jawaid M. Mechanical properties of kenaf fibre reinforced polymer composite: A review. Constr Build Mater [Internet]. 2015;76:87-96. Available from: http://dx.doi. org/10.1016/j.conbuildmat.2014.11.043

12. Awoyera PO, Adesina A. Plastic wastes to construction products: Status, limitations and future perspective. Case Stud Constr Mater [Internet]. 2020;12:e00330. Available from: https://doi. org/10.1016/j.cscm.2020.e00330

13. Pang YX, Jia DM, Hu HJ, Hourston DJ, Song M. Effects of a compatibilizing agent on the morphology, interface and mechanical behaviour of polypropylene/poly(ethylene terephthalate) blends. Polymer (Guildf). 2000;41(1):357-65.

14. Francioso V, Moro C, Castillo A, Velay-Lizancos M. Effect of elevated temperature on flexural behavior and fibers-matrix bonding of recycled PP fiber-reinforced cementitious composite. Constr Build Mater [Internet]. 2021;269(xxxx):121243. Available from: https://doi.org/10.1016/j.conbuildmat.2020.121243

15. Cardfelt A. Model system study of recycled polyethylene terephthalate and polypropylene blends by. 2015;(162).

16. Mahendra IP, Wirjosentono B, Tamrin, Ismail H, Mendez JA, Causin V. The influence of maleic anhydride-grafted polymers as compatibilizer on the properties of polypropylene and cyclic natural rubber blends. J Polym Res. 2019;26(9).

17. Akshaya EM, Palaniappan R, Sowmya CF, Rasana N, Jayanarayanan K. Properties of Blends from Polypropylene and Recycled Polyethylene Terephthalate using a Compatibilizer. Mater Today Proc [Internet]. 2020;24:359-68. Available from: https://doi.org/10.1016/j.matpr.2020.04.287

18. Matias ÁA, Lima MS, Pereira J, Pereira P, Barros R, Coelho JFJ, et al. Use of recycled polypropylene/poly(ethylene terephthalate) blends to manufacture water pipes: An industrial scale study. Waste Manag. 2020;101:250-8.

19. Wu J, Chen T, Luo X, Han D, Wang Z, Wu J. TG/FTIR analysis on co-pyrolysis behavior of PE, PVC and PS. Waste Manag [Internet]. 2014;34(3):676-82. Available from: http://dx.doi.org/10.1016/j.wasman.2013.12.005

20. Wu MH, Wang CC, Chen CY. Chemical modification of atactic polypropylene and its applications as a crystallinity additive and compatibility agent. Polymer (Guildf) [Internet]. 2020;194(March):122386. Available from: https://doi.org/10.1016/j.polymer.2020.122386

21. Hidayah Marzuki N, Irfiani N, Uzir Wahit M, Othman N, Izyan Syazana Mohd Yusoff N. Mechanical properties of kenaf fiber and montmorillonite reinforced recycled polyethylene terephthalate/recycled polypropylene. Mater Today Proc [Internet]. 2018;5(10):21879-87. Available from: https://doi.org/10.1016/j.matpr.2018.07.046

22. da Costa HM, Ramos VD, de Oliveira MG. Degradation of polypropylene (PP) during multiple extrusions: Thermal analysis, mechanical properties and analysis of variance. Polym Test. 2007;26(5):676-84.

23. López Gayarre F, Suárez González J, Blanco Viñuela R, López-Colina Pérez C, Serrano López MA. Use of recycled mixed aggregates in floor blocks manufacturing. J Clean Prod. 2017;167:713-22.

24. Cely MM, Castellar O. G, Pereira C. J, Ángel V. R. Efecto de la velocidad de calentamiento sobre las propiedades mecánicas y resistencia a la corrosión de aleaciones de titanio modificadas. Ingeniare Rev Chil Ing. 2018;26(4):577-84.

25. Wan C, Sun G, Gao F, Liu T, Esseghir M, Zhao L, et al. Effect of phase compatibility on the foaming behavior of LDPE/HDPE and LDPE/PP blends with subcritical CO2 as the blowing agent. J Supercrit Fluids [Internet]. 2017;120:421-31. Available from: http://dx.doi.org/10.1016/j.supflu.2016.05.038 